\title{
Post-Embedding Immunogold Detection of GABA-Labeling Using Large Area, High Resolution SEM Backscatter Imaging
}

\author{
Cheryl Clarkson ${ }^{1}$, Christine A. Brantner ${ }^{1}$, Nancy W. Rizzo ${ }^{2}$, Christopher T. Zugates ${ }^{3}$ and \\ Anastas Popratiloff ${ }^{1}$ \\ 1. George Washington University Nanofabrication and Imaging Center, Washington, DC, USA. \\ 2. Leica Microsystems, Buffalo Grove, USA. \\ 3. Arivis AG, Washington, DC, USA.
}

GABA ( $\gamma$-aminobutyric acid) is the most ubiquitous inhibitory neurotransmitter in the brain, present at high concentrations at synapses. Antibodies against GABA were developed more than two decades ago, providing useful utility in defining inhibitory neuronal populations and their synaptic architecture. In electron microscopy (EM) studies, anti-GABA immunolabeling has been applied using both pre- and post-embedding techniques, with the later offering a more quantifiable approach. EM offers the possibility of exploring the full spectrum of cellular and tissue morphology and pathology. However, until recently the majority of subcellular structural analyses required transmission electron microscopy (TEM) from ultrathin sections. TEM imaging imposes a sample size limitation on the complex logistics of maintaining orientation and keeping track of the overall tissue context. For many of these reasons, correlative light electron microscopy (CLEM) was developed, aiming to link the general tissue context seen in light microscopy with the small area, high-resolution information in a TEM section. The architecture of the scanning electron microscope (SEM) allows imaging of much larger samples, but the typical elastic backscatter electron signal is a challenge for biological samples that do not contain a large amount of heavy metal staining. New improvements in SEM including; sample logistics [1], stable and monochromatic field emission electron sources, solid-state detectors, broaden the spectrum of applications including neuroscience research performed in an SEM. "Block-face" imaging, $120 \mathrm{~nm}$ resin section on a Si wafer, in the SEM with elastically backscattered electrons produces inverted TEM images.

To determine the difference in GABA expression/labeling in the brainstem between epoxy and Lowicryl embedding media (Fig 1), experiments were performed with P7 old WT mice (C57BL6N). Mice were deeply anesthetized with Ketamine/Xylazine mix and perfused through the heart with a solution of 4\% PFA and $0.5 \% \mathrm{GA}$ in $0.12 \mathrm{M} \mathrm{PB} \mathrm{pH} \mathrm{7.2,} \mathrm{after} 90$ minutes of postfixation brains were washed $3 \times 5$ min with PB and vibratome sectioned (200 $\mu \mathrm{m}$ thickness). In three animals, brainstems were processed following the protocol described by Chiaramello et al. for regular epoxy embedding, while another three animal brainstem sections containing hypoglossal nucleus and spinal trigeminal nucleus were cryofixed using a Leica EM ICE high pressure freezer, Next the samples were freeze substituted at low temperature following the Rizzo et al. protocol. Immunogold experiments were paired for epoxy and lowicryl embedded sections following the protocol described by Clarkson et al. For epoxy resin embedded ultrathin sections $(120 \mathrm{~nm})$, an additional step was performed to double quench osmium from the surface using $1 \%$ sodium metaperiodate in distilled water solution $(10 \mathrm{~min})$ before the immunolabeling. Incubation of ultrathin sections in the primary antibody anti-GABA rabbit polyclonal was performed for $2 \mathrm{hr}$ at $37^{\circ} \mathrm{C}$ (humid chamber) using dilutions of 1:500 to 1:1000. GABA antibody was labeled with anti-rabbit IgG-gold conjugated secondary antibody $10 \mathrm{~nm}$, at 1:20 for $1 \mathrm{hr}$ 
$\left(37^{\circ} \mathrm{C}\right)$. Immunogold-labeled sections were observed with a FEI Helios NanoLab ${ }^{\mathrm{TM}} 660$ FIBSEM, - a field emission SEM equipped with a concentric ring backscatter detector (CBS). A CBS detector inserted below the nose piece is used with the microscope in immersion mode producing images showing dramatic improvement in the signal compared to traditional backscatter detectors positioned in the column of the SEM. At eucentric stage height, the detector receives a much stronger signal than its counterparts positioned in the column or lens. To minimize the charging artifacts, large ultrathin sections $(120 \mathrm{~nm}, 5 \times 3 \mathrm{~mm})$ of the entire brainstem were placed on semiconductor grade Si-wafer. Using FEI MAPS software, the image acquisition is managed by creating tiles in layers, we then stitch the entire area of the sample at low magnification (600x), and this image is used as a navigation map. We focus the field of view precisely at the area of the hypoglossal nucleus based on characteristic cytoarchitectural features. High-resolution $(80,000 \mathrm{x}, 1.6 \mathrm{~nm}$ pixel) imaging is performed and adjacent fields of view are stitched. High-resolution image sets are linked to the low-resolution map allowing for precise localization of features. Image data was manually segmented in Arivis software and gold particles counted by an algorithm [5].

\section{References:}

[1] JC Tapia et al, Nature Protocols 7 (2012), p. 193.

[2] A Chiaramello, M Uittenbogaard and CA Brantner, Cell Death and Disease accepted (2018).

[3] C Clarkson, FM Antunes ME and Rubio, J Neurosci 36(39) (2016) p.10214.

[4] NW Rizzo et al, J of Microscopy 263(2) (2015) p.142.

[5] The authors acknowledge funding from NICHHD DC-IDDRC (U54HD090257) and the Program for Pathology, Developmental Origins, and Prevention of Pediatric Dysphagia (P01HD083157).

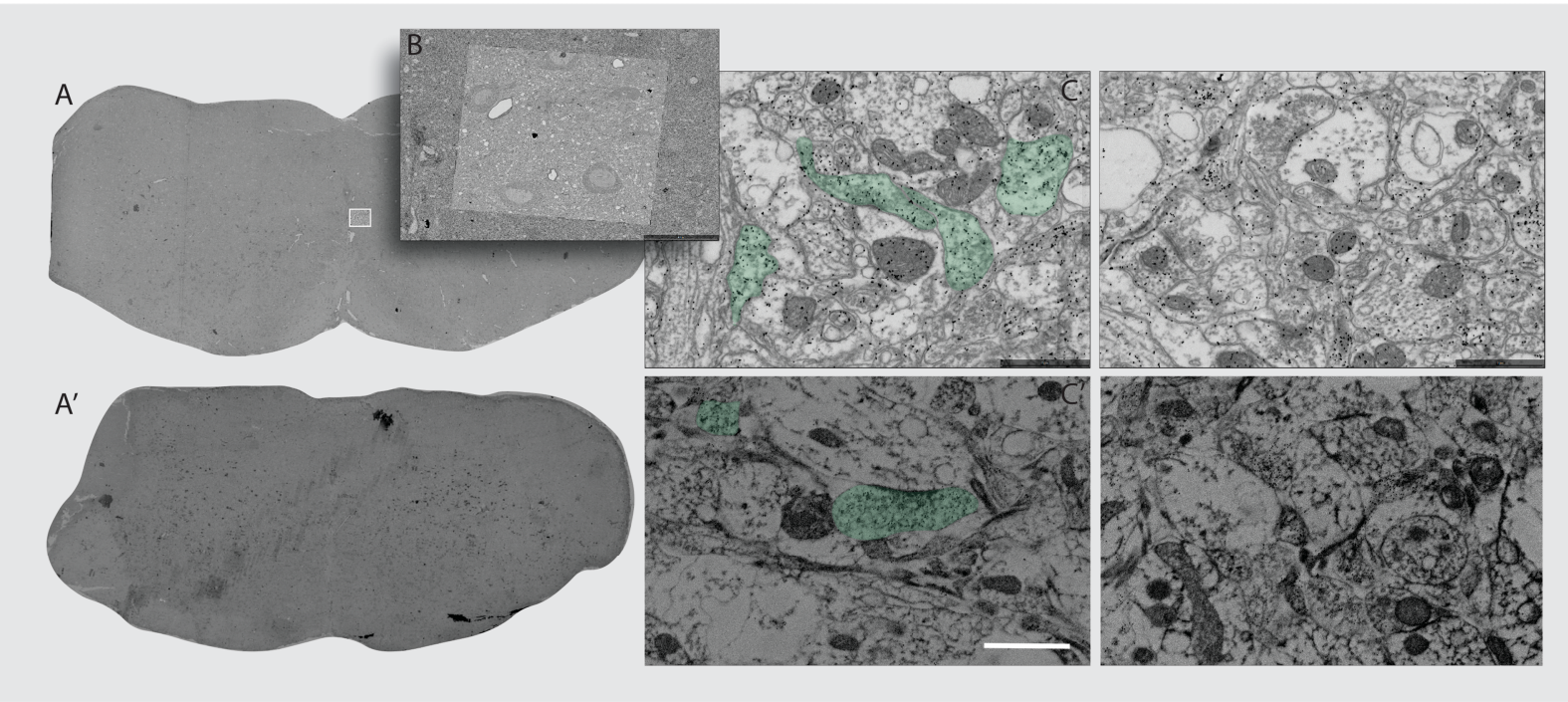

Figure 1. Left panels illustrates an entire brainstem overview (low mag 650x) for epoxy (A) and lowicryl (A') embedded sections. B panel shows a large area/high resolution tile set $(80,000 \mathrm{x})$ of the hypoglossal motor neurons located close to the central canal. Right panels show examples of positive GABA terminal endings labeled by gold particles in epoxy (C) and lowicryl (C') embedded sections. Scale bar $1 \mu \mathrm{m}$ 